\title{
Comprehensive Analysis of Electric Field Characteristics for Multi-Winding Medium Frequency Transformer
}

\author{
Pei Huang ${ }^{1,2, *}$, Renjun Dian ${ }^{1,2}$, Peng Wang ${ }^{1,2}$, Dan Wang ${ }^{3}\left(\mathbb{D}\right.$, Zhenxing Liu ${ }^{1,2}$ and Qi Wang ${ }^{1,2}$ \\ 1 School of Information Science and Engineering, Wuhan University of Science and Technology, \\ Wuhan 430081, China; dianrenjun@wust.edu.cn (R.D.); wangp1636@163.com (P.W.); \\ zhenxingliu@wust.edu.cn (Z.L.); wangqi0403043@wust.edu.cn (Q.W.) \\ 2 Engineering Research Center for Metallurgical Automation and Measurement Technology of Ministry of \\ Education, Wuhan University of Science and Technology, Wuhan 430081, China \\ 3 School of Electrical and Electronic Engineering, Huazhong University of Science and Technology, \\ Wuhan 430074, China; wangdan@mail.hust.edu.cn \\ * Correspondence: huangp@wust.edu.cn
}

check for

updates

Citation: Huang, P.; Dian, R.; Wang, P.; Wang, D.; Liu, Z.; Wang, Q. Comprehensive Analysis of Electric Field Characteristics for

Multi-Winding Medium Frequency Transformer. Energies 2021, 14, 3285 https://doi.org/10.3390/en14113285

Received: 6 May 2021

Accepted: 30 May 2021

Published: 4 June 2021

Publisher's Note: MDPI stays neutral with regard to jurisdictional claims in published maps and institutional affiliations.

Copyright: (c) 2021 by the authors. Licensee MDPI, Basel, Switzerland. This article is an open access article distributed under the terms and conditions of the Creative Commons Attribution (CC BY) license (https:// creativecommons.org/licenses/by/ $4.0 /)$.

\begin{abstract}
The multi-winding medium frequency transformer (MMFT) has attracted widespread attention, since its application in power electronic transformers can simplify the system structure and reduce the volume and weight. However, the special working conditions of MMFT due to high voltage and high power density increase the difficulty of insulation design for MMFT. For this issue, this paper presents a comprehensive analysis of electric filed characteristics for MMFT. First, the electric field model of MMFT is established using the 2-D finite element method. Based on it, the influences of connection mode, core structure, and hollow winding on the electric field characteristics of MMFT are studied, including the overall electric field distribution, maximum electric field intensity, and the electric field intensity along a fixed path. The results show that there are differences in the maximum electric field intensity for different connection modes and different core structures, which provides references for MMFT insulation design. The proposed modeling method and analysis results in this paper are important for insulation improvement of MMFT.
\end{abstract}

Keywords: medium frequency transformer; electric field; finite element method; insulation design; connection mode; core structure; hollow winding

\section{Introduction}

With the continuous development of distributed renewable energy [1], energy storage, and electric vehicles in the power grid, AC/DC hybrid distribution technology based on power electronic transformers has been rapidly developed, and among them, the multi-port power electronic transformer (M-PET) [2-5] can realize the flexible control of the power of multiple ports, and multi-input and multi-output can be achieved at the same time, which is very suitable for renewable energy grid-connected applications.

Multi-winding medium frequency transformer (MMFT) [6-9], responsible for voltage transformation and potential isolation, is the core component of M-PET. Compared with the scheme of using several single-input single-output medium frequency transformers (MFT) [10-17] to achieve multi-input and multi-output, the MMFT scheme can reduce the number of transformer and the number of voltage conversion, therefore, the system structure can be simplified, volume and weight can be reduced, and lower cost can be achieved.

The electromagnetic characteristics analysis of MMFT is more difficult due to the increase of winding number. Different from MFT, the connection mode of multiple output windings of MMFT varies with the application requirements, and the influence of connection modes should be considered for MMFT design. Besides, the number of conductors increases rapidly due to the increase of winding number, which poses a challenge to the modeling of MMFT since a large amount of computing resources and time will be 
consumed as the complexity of model increases. Previous work on MMFT can be found in [6-9]. Ref. [6] studies the accurate copper loss analysis of a three-winding transformer, taking into account the variations of phase shift, duty ratio, and amplitude of waveforms. Ref. [7] proposes a design method of shell-type MMFT in DC-DC converter, and several geometrical parameters are optimized. Ref. [8] discusses three important factors in the design process of MMFT for M-PET, involving the core material selection, wire selection, and winding placement on the core. Ref. [9] presents 7 design schemes and evaluates them in terms of efficiency, volume, leakage inductance, and parasitic capacitance. Ref. [10] presents a novel computer-aided optimal design for MFT using the non-dominated sorting genetic algorithm II. Ref. [11] proposes a design methodology to include the Litz wire configuration in the optimization process. Most previous works on MFT mainly focus on the overall design of transformer, and the analysis of electric field is insufficient.

Reliable insulation design is critical for transformers, since insulation failures of transformers not only cause power outages, but also result in costly maintenance and replacement costs. The insulation stress of M-PET is mainly concentrated on MMFT, and M-PET is usually working in high voltage conditions since they are usually connected with a power grid. Compared with a traditional power transformer, the large reduction of MFT volume brings challenges to the insulation design since it is difficult to meet high insulation requirements in a small volume. The insulation design of transformers is concerned in [18-23]. Ref. [18] designs the insulation structure of a dry-type highvoltage transformer considering volume and insulation performance. Ref. [19] focuses on transformer design with high insulation requirements, a single-layer winding is employed to eliminate insulation stress between different layers. Ref. [20] discusses the technical challenges and trade-offs on MFT design, and concludes that the power density of MFT can be improved using stronger dielectric materials for insulation. Ref. [21] proposes a novel insulation structure for MFT with high insulation level, the primary winding is impregnated in cast epoxy resin and especially enhanced at the terminal. Ref. [22] proposes a novel cooling and insulation structure based on the parallel-concentric winding concept. Ref. [23] carries out a study of the parameters that affect the breakdown voltage in the insulation supports of a standard dip and bake dry-type transformer. As can be seen, previous studies mainly focus on improving the insulation structure and winding structure to meet the high insulation requirements.

Compared with previous work on MMFT design, the novelty of this paper lies in the fact that it provides a new perspective to improve insulation performance and that several important factors that affect the electric field characteristics are analyzed, including connection mode, core structure, and hollow winding, which provide a reference for MMFT insulation design.

The main contributions of this paper include: (a) the influences of different connection modes on the electric field distribution of MMFT are studied, involving series connection mode and independent operation mode. (b) the influences of different core structures on the electric field distribution of MMFT are studied in detail, including the overall electric field distribution, maximum electric field intensity, and the electric field intensity along fixed path. Two kinds of core structures are common for MFT, involving shell type and core type [21]. (c) the effects of hollow windings on the electric field distribution of MMFT are studied in detail. The hollow windings have been considered in MFT design, and thermal analysis of MFT with hollow windings is presented in [24]. It has been identified as a promising solution to quickly absorb the heat dissipated from the conductors that placing phase change material inside hollow conductors [25], and hollow windings have been used for direct cooling in electrical machine [26]. The finite element method [27] is the most commonly used method for electromagnetic field analysis of transformer [28], and 2-D finite element method is chosen in this paper for electric field calculation and analysis of MMFT. 
This paper presents the electric field modeling and analysis for MMFT, and the relationship between the sections is as follows: The basic principle of electric field calculation using finite element method is presented in Section 2, and the electric field model of MMFT is established in Section 3. Sections 4-6 present the electric field analysis for different connection modes, different core structures, and hollow windings with different hollow ratios. The electric field calculation principle and electric field model in Sections 2 and 3 lay a basis for electric field analysis in Sections 4-6.

\section{2-D Electric Field Calculation Method}

The basic principle of electric field calculation using finite element method is presented in this section. The electric field calculations for different connection modes, different core structures and hollow windings with different hollow ratios are carried out in Sections 4-6, though the transformer structure or parameters are different, the principles of electric field calculation are the same.

The basic principle of finite element method is to divide the whole field with finite units, and the potential of each point is determined by making the electrostatic field energy of the whole field as the minimum [29]. The analysis object is taken as a combination of a finite number of units with simple shapes, and the properties within the unit are approximated by a simple function as follow:

$$
\Phi=\alpha_{1}+\alpha_{2} x+\alpha_{3} y
$$

Usually, the potential energy $X$ in a 2-D field can be expressed by the following formula:

$$
X=\frac{1}{2} \iint \varepsilon\left\{\left(\frac{\partial \Phi}{\partial x}\right)^{2}+\left(\frac{\partial \Phi}{\partial y}\right)^{2}\right\} d x d y
$$

where $\varepsilon$ is dielectric constant. The nodal potential can be solved by minimizing the potential energy of the whole field. To minimize $X$, the derivative of $X$ with respect to the potential at each node can be set to zero, i.e.,

$$
\frac{\partial X}{\partial \Phi_{i}}=\sum \frac{\partial X(e)}{\partial \Phi_{i}}=0
$$

\section{2-D Electric Field Modeling for MMFT}

The case study in this paper was conducted on a $1 \mathrm{MW} / 20 \mathrm{kHz}$ MMFT in a DCDC converter [7] as shown in Figure 1. There are two connection modes for the output windings, one is series connection mode as shown in Figure 1a, and the other is independent operation mode as shown in Figure 1b. This MMFT consists of two primary windings and twelve secondary windings, and the specifications and parameters of this MMFT [7] are shown in Table 1. Two kinds of core structures for this MMFT are studied in this paper, including shell type and core type, as shown in Figure 2. The primary winding is the low voltage winding (LV winding), and the secondary winding is the high voltage winding (HV winding). For shell type, the primary and secondary windings go around the same core column. For core type, the primary and secondary windings go around two core columns. 


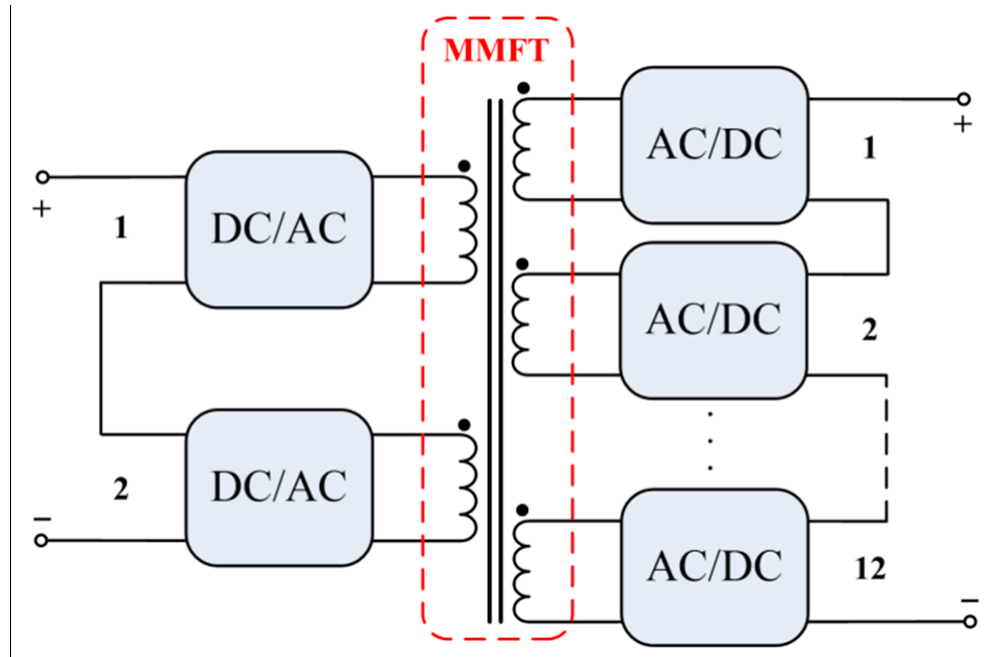

(a)

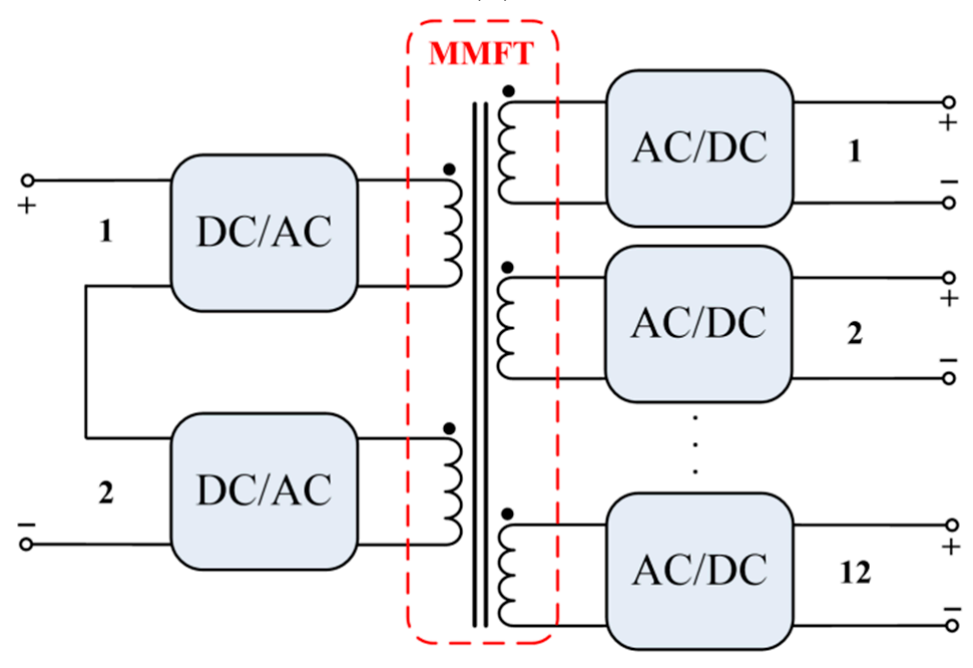

(b)

Figure 1. The schematic diagram of DC-DC converter using MMFT with different connection modes: (a) Series connection mode; (b) Independent operation mode.

Table 1. Specifications and parameters of this MMFT.

\begin{tabular}{ccc}
\hline Parameter & Symbol & Value \\
\hline Power & $P$ & $1 \mathrm{MW}$ \\
Working frequency & $f$ & $20 \mathrm{kHz}$ \\
Primary voltage & $U_{1}$ & $3 \mathrm{kV}$ \\
Secondary voltage & $U_{2}$ & $30 \mathrm{kV}$ \\
Rms primary current & $I_{1}$ & $333.33 \mathrm{~A}$ \\
Rms secondary current & $I_{2}$ & $33.33 \mathrm{~A}$ \\
Number of primary winding & $m$ & 2 \\
Number of secondary winding & $n$ & 12 \\
Number of turns for primary winding (total) & $N_{1}$ & 18 \\
Number of turns for secondary winding (total) & $N_{2}$ & 180 \\
\hline
\end{tabular}




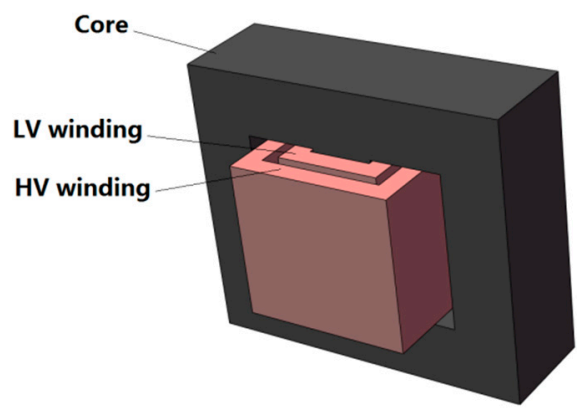

(a)

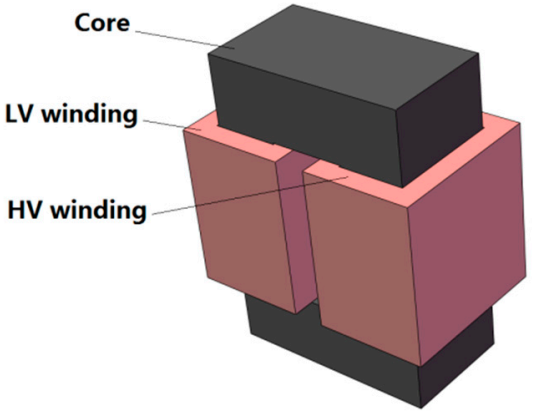

(b)

Figure 2. MMFT with different core structures. (a) Shell type. (b) Core type.

In order to save the computational resources and improve the simulation efficiency, $1 / 2$ model was adopted. The structure of this MFT is symmetrical up and down, and the high voltage is concentrated in the upper half of windings, $1 / 2$ model is applicable by adding symmetric boundary condition. Therefore, the number of conductors is greatly reduced, and so are the simulation resources and time. Electric field models are established in finite element simulation software ANSYS Electromagnetics/Maxwell 16.0. Figure 3a,b provide the winding arrangement for shell type and core type, respectively. The primary winding is the low voltage winding ( $\mathrm{LV}$ winding), and the secondary winding is the high voltage winding ( $\mathrm{HV}$ winding). There are 12 secondary windings, and there are 15 turns for each secondary winding. There are 2 rows for each secondary winding, and there is a vacancy in the second row. In Figure 3a, both primary and secondary winding go around the middle core column, therefore, the vacancy appears on the right of the second row. In Figure $3 b$, the primary winding goes around the left core column while the secondary winding goes around the right core column, therefore, the vacancy appears on the left of the second row. Table 2 provides the model parameters of core and windings.

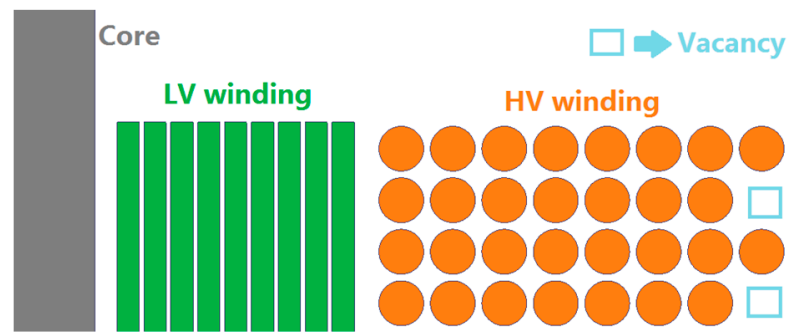

(a)

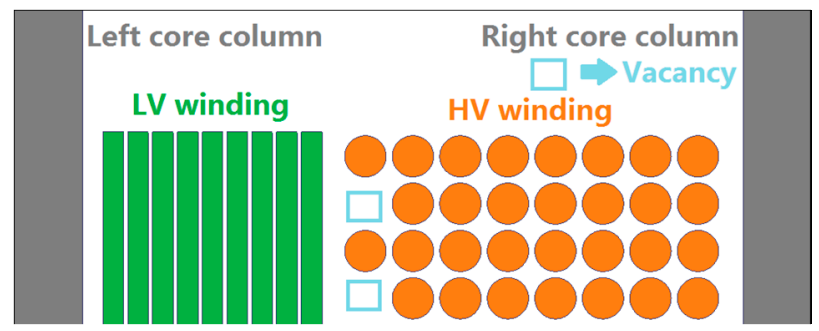

(b)

Figure 3. Winding arrangements for different core structures: (a) Shell type; (b) Core type. 
Table 2. Parameters of core and windings for this MMFT.

\begin{tabular}{ccc}
\hline & Parameter & Value \\
\hline \multirow{4}{*}{ Core } & Window width & $64 \mathrm{~mm}$ \\
& Window height & $149 \mathrm{~mm}$ \\
& Core stack length & $21.6 \mathrm{~mm}$ \\
Core stack width & $24.5 \mathrm{~mm}$ \\
Core stack number & 4 \\
\hline \multirow{3}{*}{ Windings } & Wire type for primary winding & Foil conductor \\
& Wire type for secondary winding & Litz wire \\
& Size of the foil conductor (length/width) & $55 \mathrm{~mm} / 2 \mathrm{~mm}$ \\
Size of the Litz wire (radius) & $2 \mathrm{~mm}$ \\
\hline
\end{tabular}

The insulation distance between primary winding layers is $0.4 \mathrm{~mm}$, the insulation distance between secondary winding layers is $0.6 \mathrm{~mm}$, and the insulation distance between primary and secondary winding is $2.2 \mathrm{~mm}$. there are nine foil conductors in primary winding, and ninety litz conductors in secondary winding. The voltage excitations for primary winding in the two connection modes are the same, decreasing from outside to inside. The voltage excitation for secondary winding decreases from top to bottom in series connection mode, and the voltage excitations for each output winding are consistent in independent operation mode.

\section{Electric Field Analysis for MMFT with Different Connection Modes}

The electric field distributions of MMFT can be obtained using the established finite element model. The core structure is shell type, and the windings are arranged as shown in Figure 3a. Two connection modes are simulated, including series connection mode and independent operation mode. In series connection mode, the excitation voltages of multiple output windings increase from bottom to top, while in independent operation mode, the excitation voltage of each output winding is consistent. The electric field distributions for the two connection modes are simulated as shown in Figure $4 \mathrm{a}, \mathrm{b}$. The maximum electric field intensities $E_{\max }$ for the two connection modes are shown in Table 3, as can be seen, $E_{\max }$ of series connection mode is higher, which is 4.8 times that of the independent operation mode. In series connection mode, the excitation voltage of multiple output windings is cumulative, resulting in a high voltage and a high electric field intensity in the first output winding.

Table 3. Maximum electric field intensities for different connection modes.

\begin{tabular}{cc}
\hline Connection Mode & $E_{\max }(\mathrm{V} / \mathrm{m})$ \\
\hline Series connection mode & $1.9432 \times 10^{7}$ \\
Independent operation mode & $4.0876 \times 10^{6}$ \\
\hline
\end{tabular}



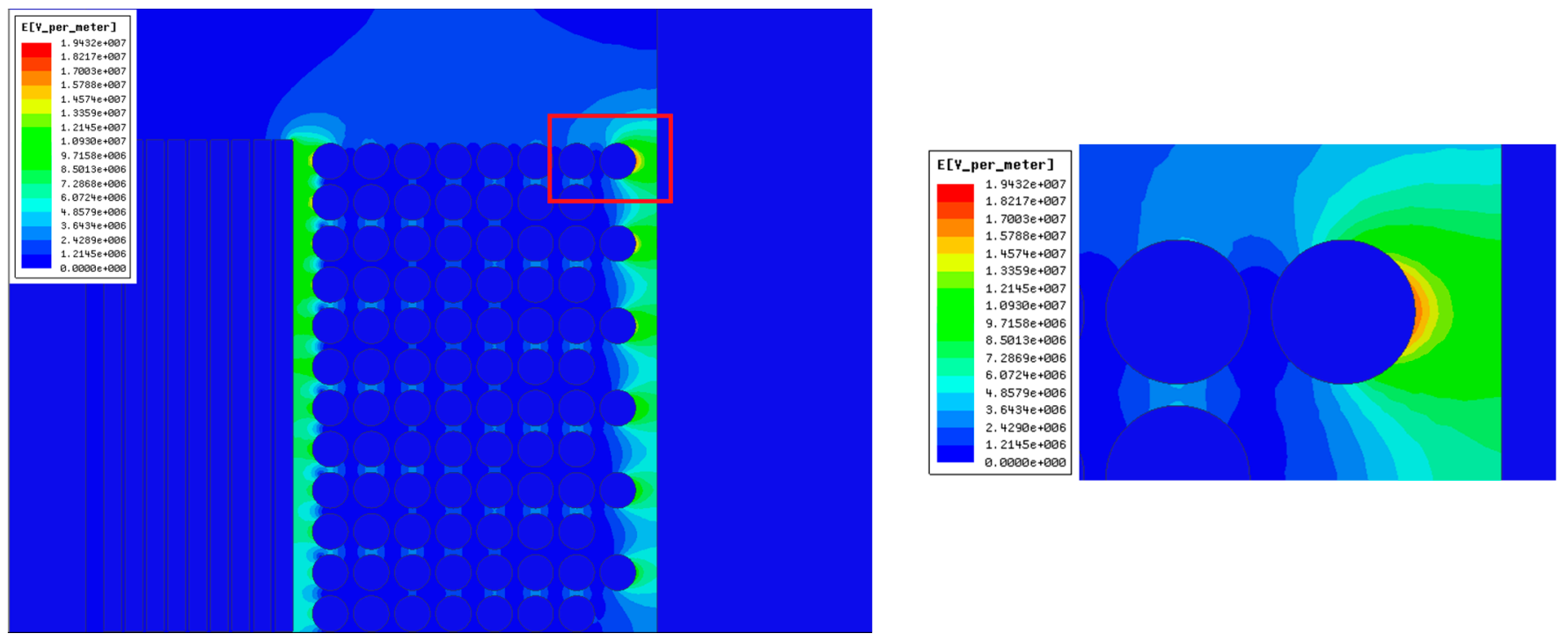

(a)
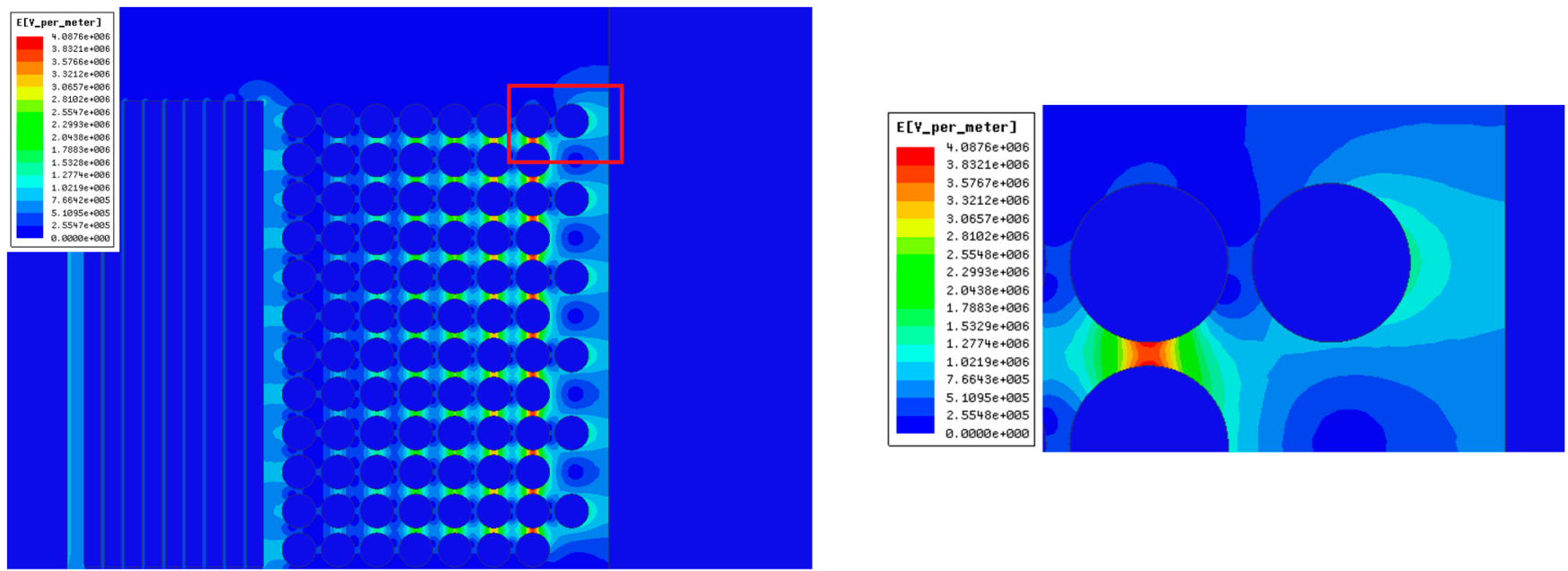

(b)

Figure 4. The electric field simulation results for different connection modes: (a) Series connection mode; (b) Independent operation mode.

\section{Electric Field Analysis for MMFT with Different Core Structures}

Using the established electric field model in Section 3, the electric field distributions for MMFT with different core structures are obtained as shown in Figure 5a,b. The connection mode is series connection. For both shell type and core type, the high electric field intensity occurs in the upper part of the secondary winding, which is mainly concentrated in the region between primary and secondary winding, and the region between the secondary winding and core. The maximum electric field intensities $E_{\max }$ for different core structures are shown in Table 4 , and as can be seen, $E_{\text {max }}$ of shell type is higher than core type, which is 1.2 times that of the core type. The change of core structure affects the arrangement of windings, and further leads to the change of electric field distribution and maximum electric field intensity. 

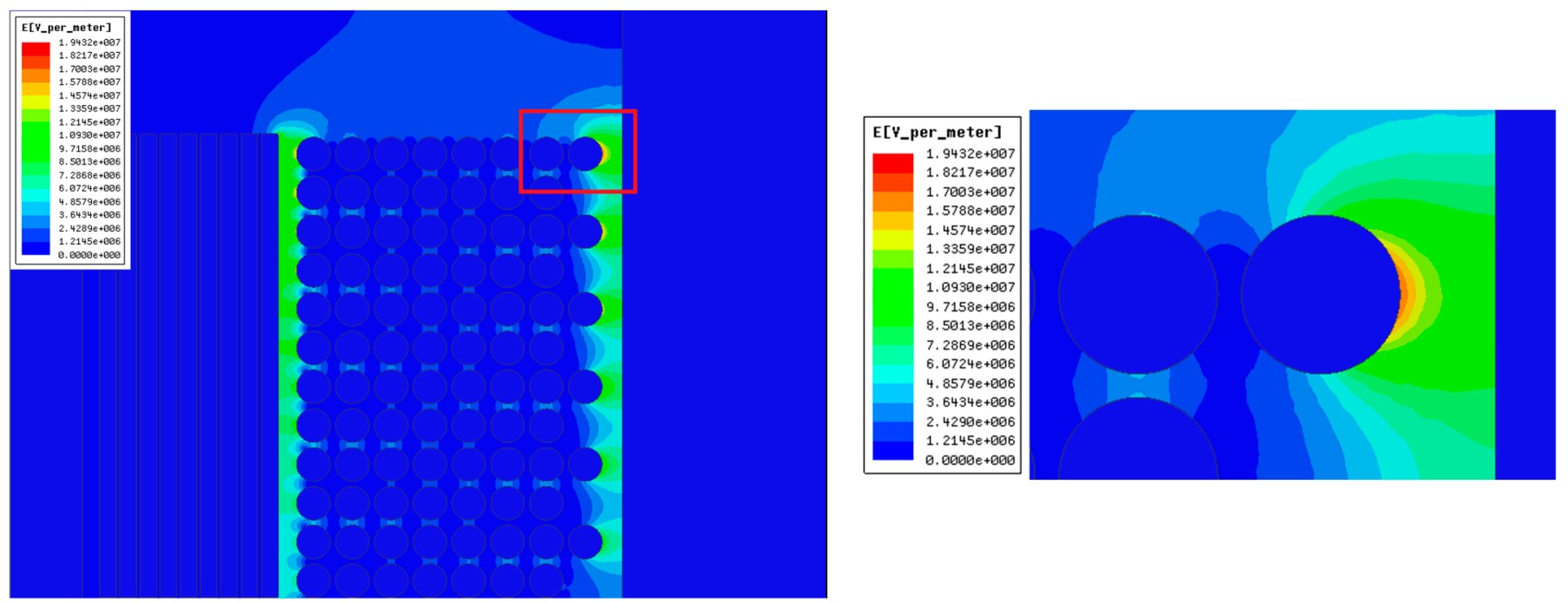

(a)
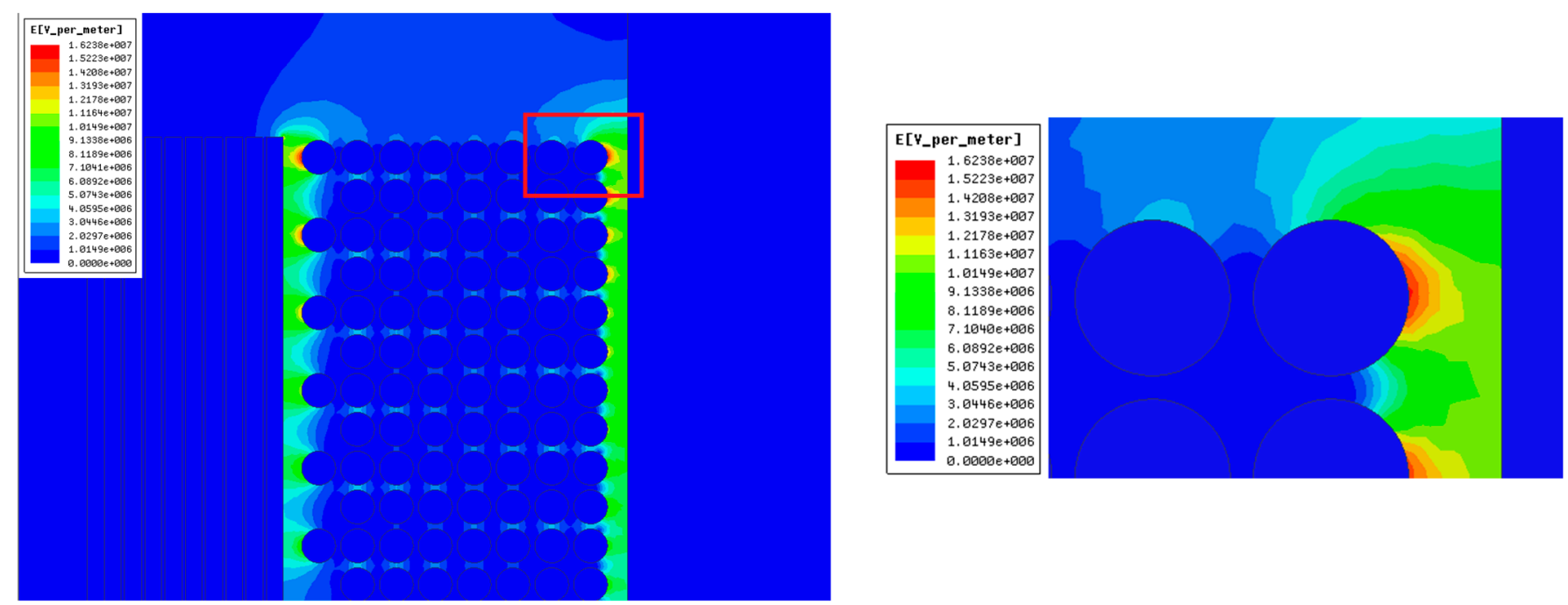

(b)

Figure 5. The electric field simulation results for different core structures: (a) Shell type; (b) Core type.

Table 4. Maximum electric field intensities for different core structures.

\begin{tabular}{cc}
\hline Core Structure & $\boldsymbol{E}_{\text {max }} / \mathrm{V} / \mathbf{m}$ \\
\hline Shell type & $1.9432 \times 10^{7}$ \\
Core type & $1.6238 \times 10^{7}$ \\
\hline
\end{tabular}

The electric field intensity distributions along predefined paths are researched. As it can be seen in Figure 5a,b, high electric field intensity arises mainly in two regions, the region between primary and secondary winding, and the region between secondary winding and core. Therefore, two paths are preset in these two regions as shown in Figure 6. Path 1 is in the region between primary and secondary windings, and $0.1 \mathrm{~mm}$ away from the secondary winding. Path 2 is in the region between secondary winding and core, and $0.1 \mathrm{~mm}$ away from the secondary winding. The upper vertices of path 1 and path 2 are aligned with the upper edge of foil conductor in primary winding, and the lengths of path 1 and path 2 are equal to the length of the foil conductor in primary winding. 


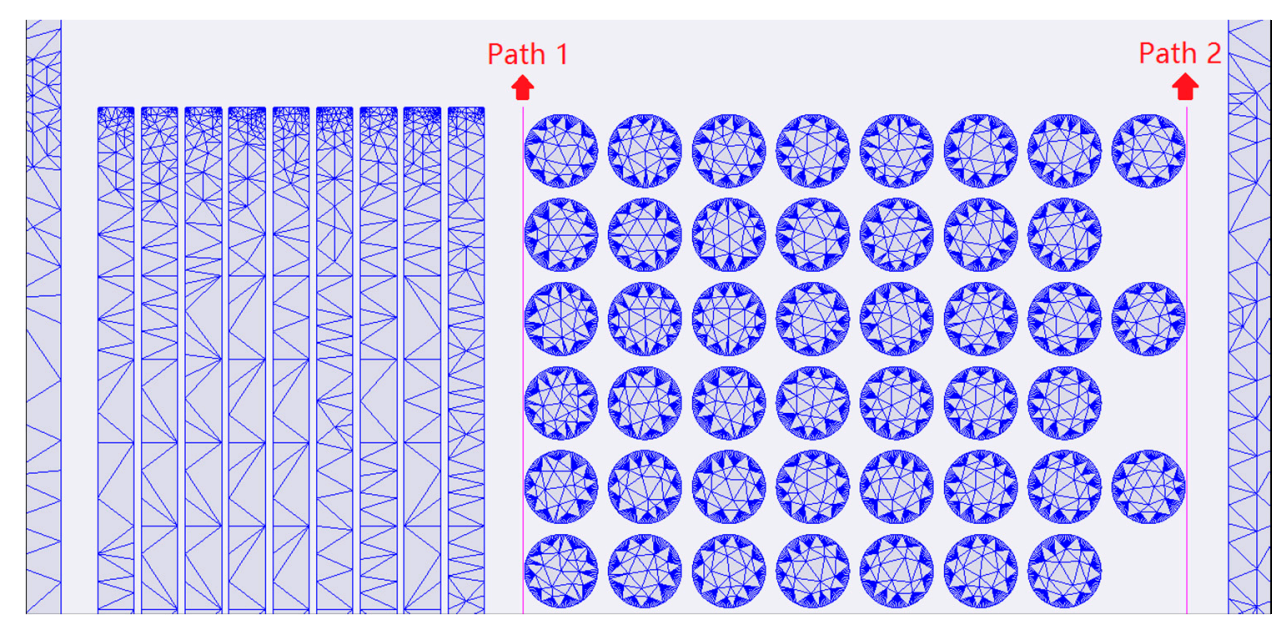

Figure 6. The predefined paths: path 1 and path 2 .

The electric field intensity distributions along fixed path can be obtained using the established finite element models as shown in Figure 5a,b. The electric field intensity distributions along path 1 and 2 for shell-type and core-type structures are shown in Figure $7 \mathrm{a}, \mathrm{b}$, respectively. The horizontal axis from $0 \mathrm{~mm}$ to $55 \mathrm{~mm}$ represents the highest point to the lowest point of the path. As can be seen, the electric field intensity fluctuates along the winding height, and the peak value decreases from top to bottom. First, for secondary winding (litz wire winding), the distance between conductor and core varies with the winding height since the shape of conductor is round and there are insulation gaps between adjacent layers, resulting in the fluctuation of electric field intensity along the winding height. Second, for series connection mode, the excitation voltage value decreases from top to bottom of the winding; therefore, the amplitude of electric field intensity decreases from top to bottom.

Path 1 is in the region between primary and secondary windings. For shell-type structure, 12 conductors in the left of secondary winding are closest to primary winding (Figure 6 shows only the upper part of the secondary winding for shell-type structure), while for core-type structure, 6 conductors in the left of secondary winding are closest to primary winding. Therefore, for path 1 , there are 12 peaks for shell-type structure, and 6 peaks for core-type structure as shown in Figure 7a.

Path 2 is in the region between secondary winding and core. For shell-type structure, 6 conductors in the right of secondary winding are closest to core, while for core-type structure, 12 conductors in the right of secondary winding are closest to core. Therefore, for path 2, there are 6 peaks for shell-type structure, and 12 peaks for core-type structure as shown in Figure $7 \mathrm{~b}$. 


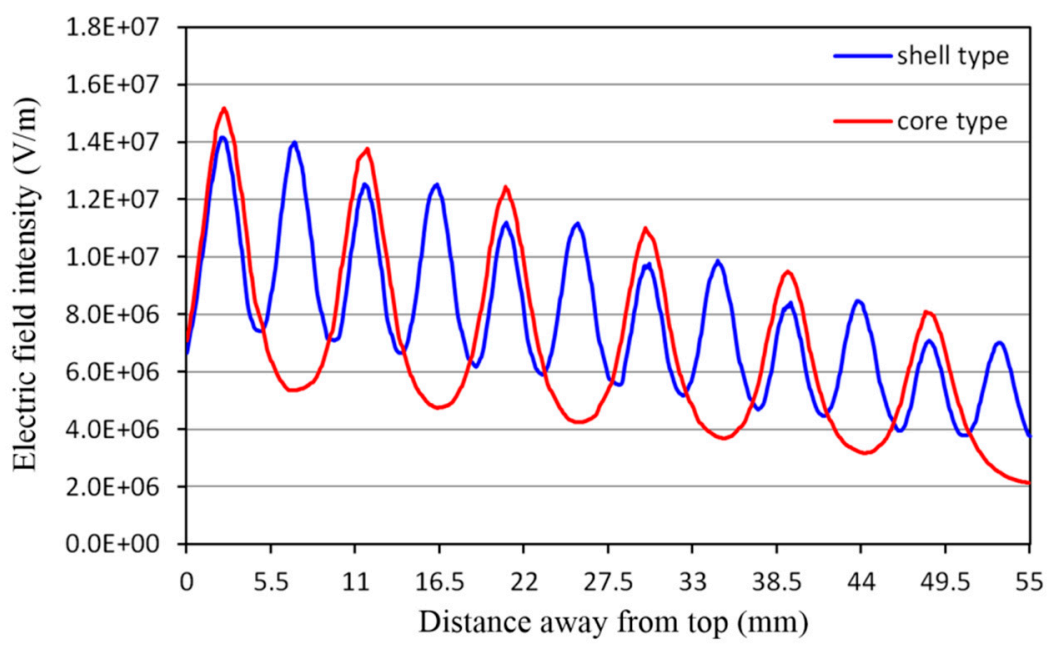

(a)

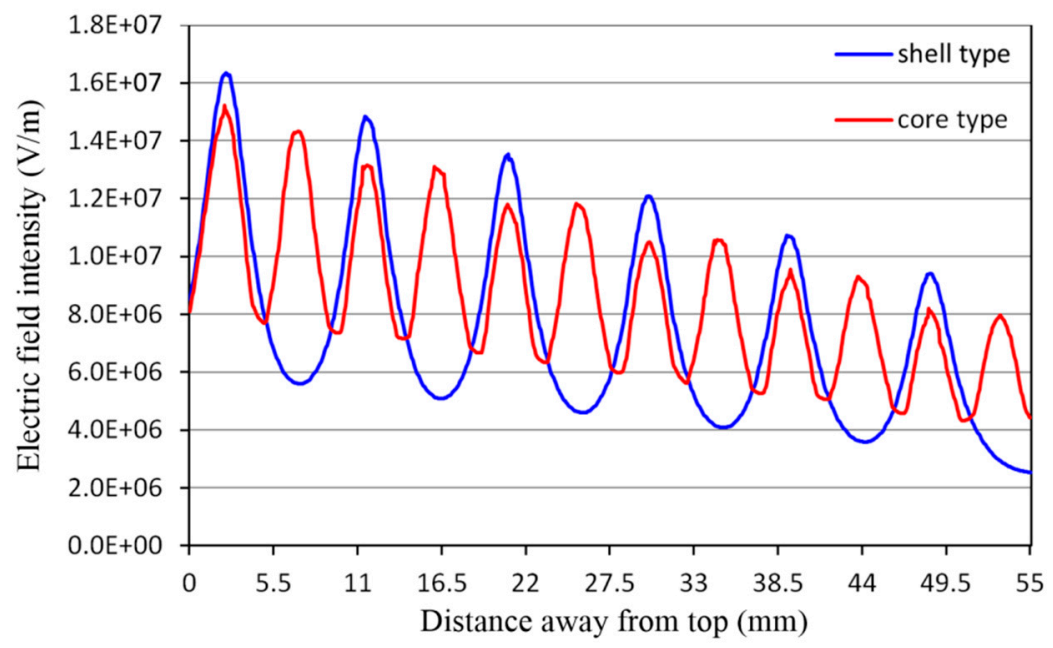

(b)

Figure 7. Electric field intensity along fixed paths for MMFT with shell-type and core-type structures: (a) path 1 ; (b) path 2 .

\section{Electric Field Analysis for MMFT with Hollow Winding}

Due to the skin effect, the current in conductor accumulates towards the conductor surface at high frequency. Compared with solid conductor, the utilization rate of winding material can be improved by using hollow winding. Besides, the cooling efficiency can be improved by introducing cooling medium into the hollow winding. In order to study the influence of hollow winding on electric field distribution, several important parameters of hollow winding should be concerned.

As shown in Figure 8, the radius of the hollow region is called the hollow radius, and is denoted by $r_{0}$. The hollow ratio $\Delta_{h}$ is an important parameter for hollow winding design, which is defined as follows:

$$
\Delta_{h}=\frac{t}{\delta}
$$

where $t$ refers to the thickness of the hollow winding as shown in Figure 8 , and $\delta$ refers to the skin depth of a winding at a specified frequency, which can be calculated as follows:

$$
\delta=\sqrt{\frac{1}{\pi f \mu \sigma}}
$$


where $f$ refers to the working frequency, $\mu$ refers to the permeability of conductor, and $\sigma$ refers to the conductivity of conductor. The values of parameters are shown in Table 5.

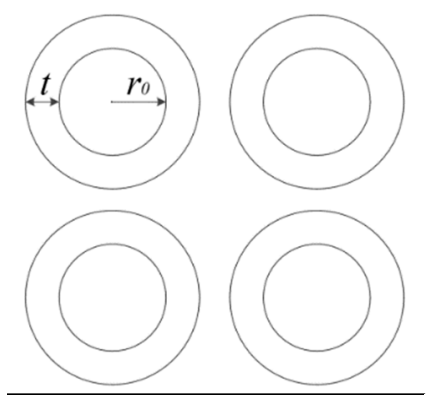

Figure 8. The parameters for hollow winding.

Table 5. The values of parameters in formula (5).

\begin{tabular}{cccc}
\hline$f / \mathbf{k H z}$ & $\mu / \mathrm{H} / \mathbf{m}$ & $\sigma / \mathrm{S} / \mathrm{m}$ & $\delta / \mathbf{m m}$ \\
\hline 20 & $4 \pi \times 10^{-7}$ & $5.8 \times 10^{7}$ & 0.4673 \\
\hline
\end{tabular}

The 2-D electric field model of MMFT with hollow winding is established in ANSYS. The core structure is shell-type, and the connection mode is series connection. The windings are arranged as shown in Figure 6, and the only difference is that the conductor of the secondary winding is hollow conductor as shown in Figure 8, and three hollow conductors with different hollow radiuses $r_{0}$ are researched, including $1.0 \mathrm{~mm}, 1.5 \mathrm{~mm}$, and $1.8 \mathrm{~mm}$. Table 6 offers the hollow radius $r_{0}$, the thickness of the hollow conductor $t$, skin depth $\Delta$, and the hollow ratio $\Delta_{h}$ of the three windings. The excitation voltage distribution of the three windings is consistent with that of the shell-type solid conductor winding in Section 3. The insulation distance between primary winding layers is $0.4 \mathrm{~mm}$, the insulation distance between secondary winding layers is $0.6 \mathrm{~mm}$, and the insulation distance between primary and secondary winding is $2.2 \mathrm{~mm}$.

Table 6. The parameters of the three hollow windings.

\begin{tabular}{ccccc}
\hline Number & $\boldsymbol{r}_{\mathbf{0}} / \mathbf{m m}$ & $\boldsymbol{t} / \mathbf{m m}$ & $\Delta / \mathbf{m m}$ & $\boldsymbol{\Delta}_{\boldsymbol{h}}$ \\
\hline $\mathrm{a}$ & 1.0 & 1.0 & 0.4673 & 2.14 \\
$\mathrm{~b}$ & 1.5 & 0.5 & 0.4673 & 1.07 \\
$\mathrm{c}$ & 1.8 & 0.2 & 0.4673 & 0.43 \\
\hline
\end{tabular}

Figure 9a-c provides the electric field distributions for the three hollow windings, and high electric field intensity arises mainly in two regions, the region between primary and secondary winding, and the region between secondary winding and core. Table 7 shows the maximum electric field intensities $E_{\max }$ of the three hollow windings, and as can be seen, $E_{\max }$ basically remains unchanged with the decrease of the hollow ratio $\Delta_{h}$, and $E_{\max }$ of hollow winding is slightly higher than that of solid conductor winding. First, the change of area of the hollow part does not affect the potential of the conductor, so the electric field intensity is basically unchanged at different hollow radiuses as shown in Table 7. Second, the potential of the conductor remains the same whether it is a solid conductor or a hollow conductor when the excitation voltage is consistent, thus, the electric field intensities of solid conductor winding and hollow winding are basically the same, and the hollow part causes the variation of the model's subdivision mesh, which may lead to slight differences of the calculation results; therefore, $E_{\max }$ of solid conductor winding and hollow winding are not exactly the same as shown in Table 7. 

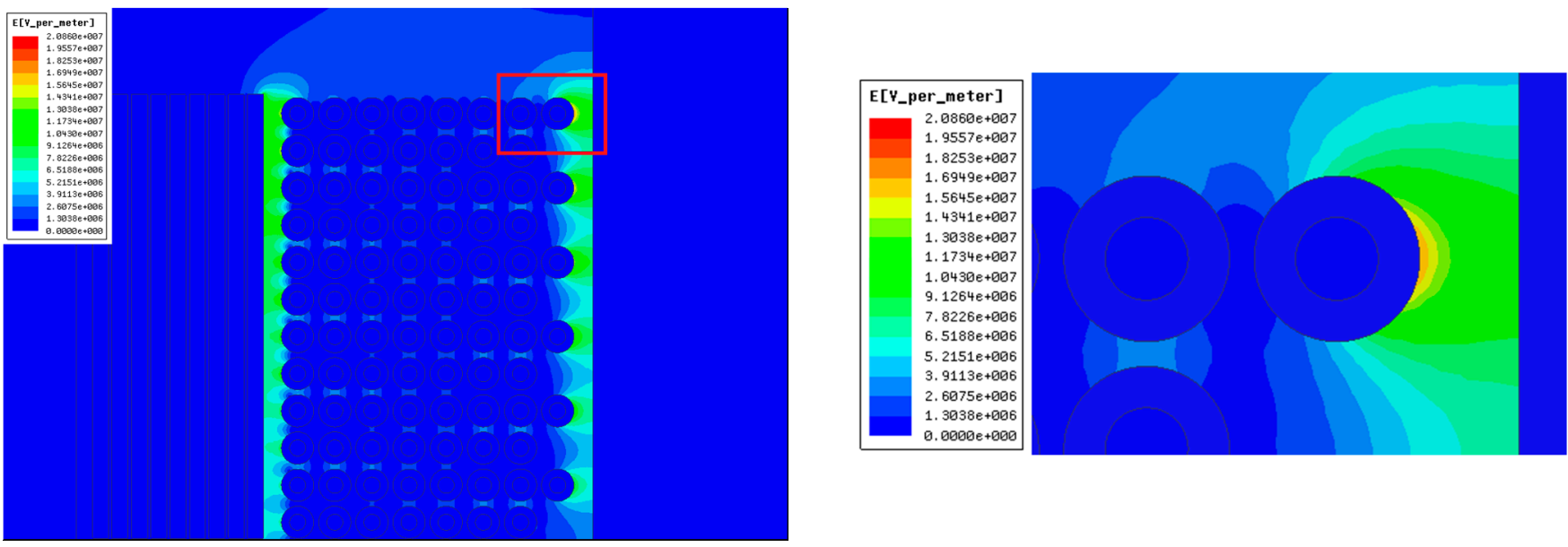

(a)
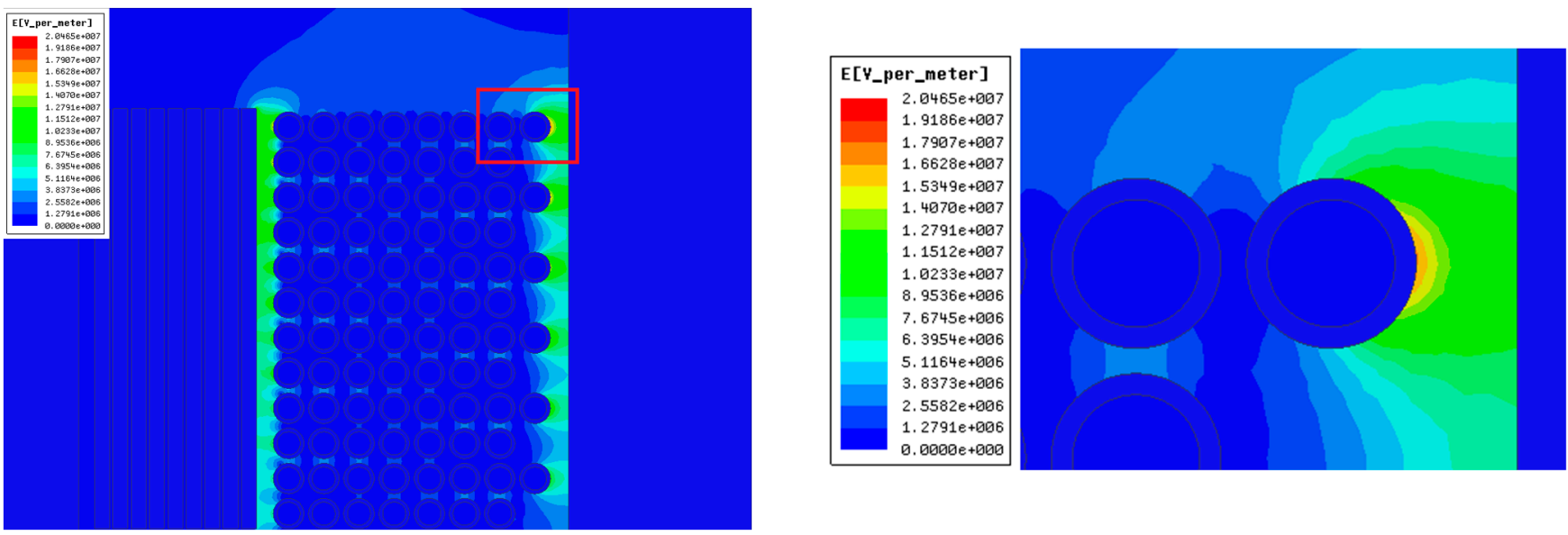

(b)
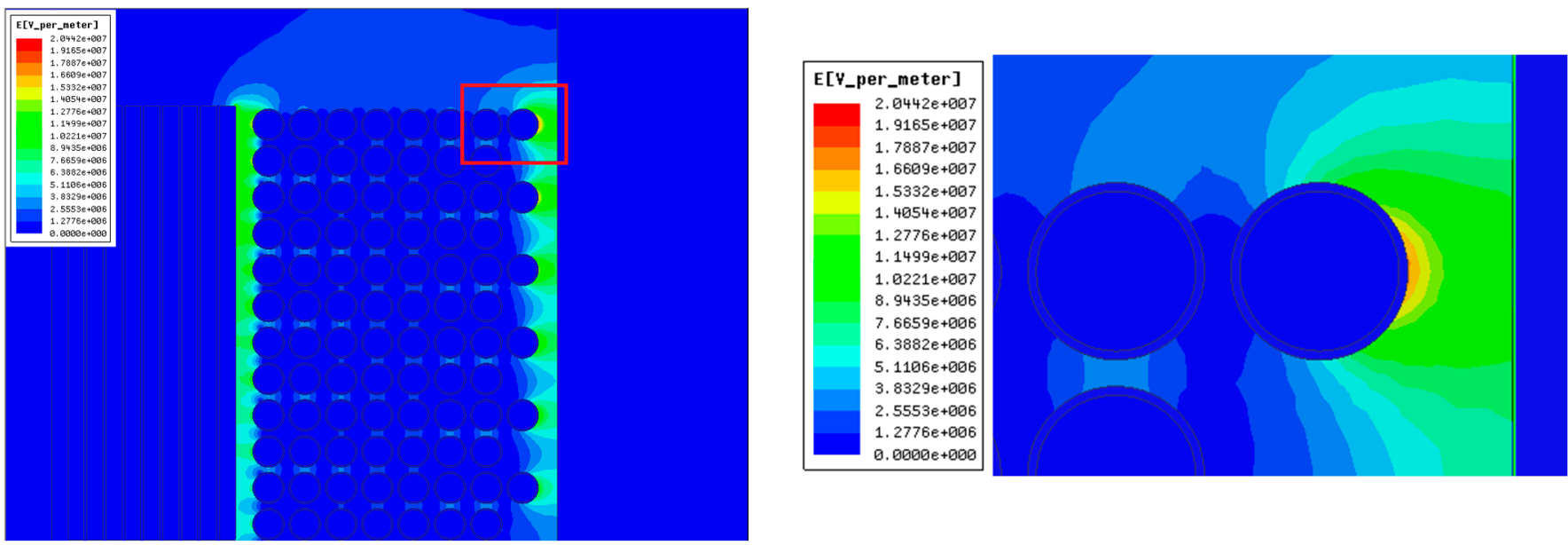

(c)

Figure 9. Electric field intensity distributions of MMFT with hollow windings for different hollow radiuses: (a) $r_{0}=1.0 \mathrm{~mm}$; (b) $r_{0}=1.5 \mathrm{~mm}$; (c) $r_{0}=1.8 \mathrm{~mm}$. 
Table 7. Maximum electric field intensities for the three hollow windings.

\begin{tabular}{cccc}
\hline Number & $\begin{array}{c}\text { Hollow Radius } \\
\boldsymbol{r}_{\mathbf{0}} / \mathbf{m m}\end{array}$ & Hollow Ratio $\boldsymbol{\Delta} \boldsymbol{h}$ & $\boldsymbol{E}_{\text {max }} /$ V/m \\
\hline $\mathrm{a}$ & 1 & 2.14 & $2.0860 \times 10^{7}$ \\
$\mathrm{~b}$ & 1.5 & 1.07 & $2.0465 \times 10^{7}$ \\
$\mathrm{c}$ & 1.8 & 0.43 & $2.0442 \times 10^{7}$ \\
$\mathrm{~d}$ & Solid conductor & Solid conductor & $1.9432 \times 10^{7}$ \\
\hline
\end{tabular}

The electric field intensity distribution along predefined path for the three hollow windings is researched. Two paths are preset, as shown in Figure 6. The electric field intensity distribution along fixed path can be obtained using the established finite element model, and the electric field intensity distributions along path 1 and 2 for the three hollow windings are shown in Figure 10a,b respectively. As can be seen, for the three hollow windings and solid conductor winding, the variation trend of electric field intensity along the winding height is consistent, and there is little difference in the magnitude of electric field intensity. First, the core structure of the three hollow windings and solid conductor winding is the same, and the number of conductors closest to primary winding is the same; thus, the number of peaks along path 1 for the four windings is the same. For the same reason, the number of peaks along path 2 is the same, as shown in Figure 10a,b. Second, as discussed in previous paragraph, the electric field intensities are basically unchanged at different hollow radiuses, and the electric field intensities of solid conductor winding and hollow winding are basically the same; therefore, the magnitudes of electric field intensity for the four windings are almost uniform as shown in Figure 10a,b.

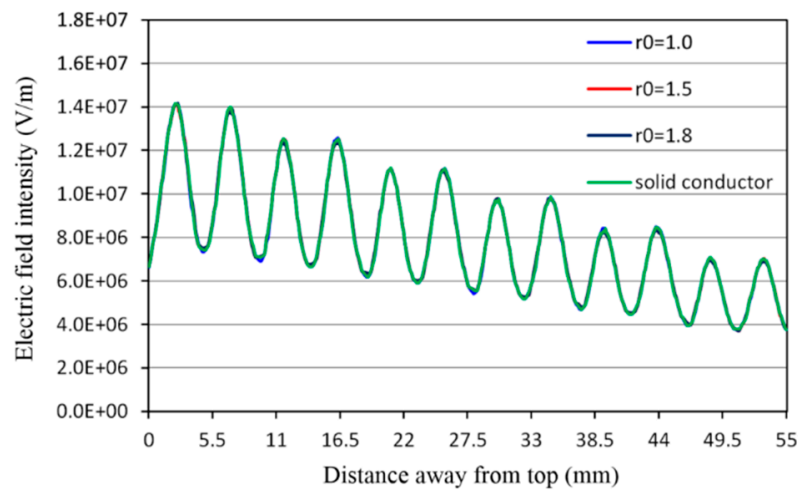

(a)

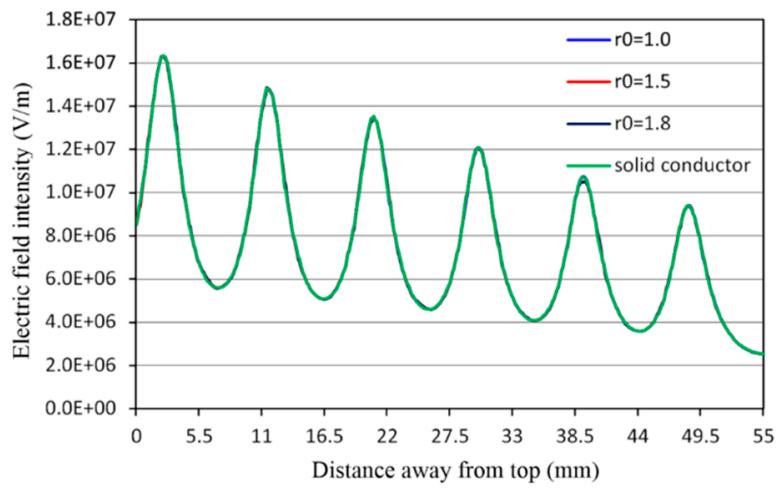

(b)

Figure 10. Electric field intensity along fixed paths for three hollow windings with different hollow radiuses: (a) path 1; (b) path 2.

\section{Discussion}

This paper presents the electric field modeling and analysis for MMFT. The electric field distributions for different connection modes, different core structures, and hollow windings with different hollow ratios are simulated and compared, and the electric field intensities along fixed paths are obtained. The results show that there are differences in the maximum electric field intensity for different connection modes and different core structures.

Compared with previous work, the feature of this paper lies in the specialized and detailed study on the electric field characteristics of MMFT. Most previous works on MFT mainly focus on the overall design of transformer, and the analysis of electric field is often insufficient-only the overall electric field distribution and maximum electric field intensity are offered, which vary with the transformer constructions, core and winding parameters, and safety factors of insulation; therefore, it is difficult to use a unified standard to compare 
the performance of electric field in different literatures. However, through the comparative analysis with the previous research results, the following two conclusions can be obtained:

(a) For the overall electric field distribution, the proportion of the area with high electric field intensity in the transformer area in this paper is much smaller than that in previous work, for example in [21]. The reduction of area with high electric field intensity is beneficial to reduce the probability of insulation failure.

(b) For the value of the maximum electric field intensity, close result can be found in previous work, for example, $14 \mathrm{kV} / \mathrm{mm}$ in [21], while the range in this paper is $6 \sim 20 \mathrm{kV} / \mathrm{mm}$, which proves that the results in this paper are at a reasonable level.

\section{Conclusions}

This paper focuses on the electric field modeling and analysis for MMFT, and the influences of connection mode, core structure, and hollow winding on the electric field characteristics are studied, the main work is as follows:

(a) The electric field model of MMFT using 2-D finite element method is established, and the overall electric field distribution, maximum electric field intensity, and the electric field intensity along fixed path can be obtained.

(b) The electric field distributions of MMFT for series connection mode and independent operation mode are simulated and compared, and the results show that series connection mode achieves higher maximum electric field intensity.

(c) The electric field distributions of MMFT with shell type and core type structures are simulated and compared; the results show that maximum electric field intensity of shell type structure is higher. The electric field intensity along paths 1 and 2 are simulated and compared, and the results show that the electric field intensity fluctuates along the winding height, the peak value decreases from top to bottom.

(d) The electric field model of MMFT with hollow winding is established, and the electric field distributions of hollow windings with different hollow ratios are simulated and compared; the results show that the change of hollow ratio has little effect on the maximum electric field intensity. Besides, there is little difference on maximum electric field intensity of hollow winding and solid conductor winding.

The proposed modeling method and research results provide a basis for insulation design of MMFT. The electric field characteristics can be improved by selecting the proper connection mode of converter and selecting the proper core structure. The proposed method and analysis results are expected to facilitate further improvements in insulation design of MFT, which is crucial for long-term service.

Author Contributions: Conceptualization, P.H. and D.W.; methodology, P.H.; software, P.H.; validation, P.H.; investigation, Q.W.; writing-original draft preparation, P.H.; writing-review and editing, D.W., R.D. and P.W.; visualization, P.W.; supervision, Z.L.; project administration, R.D.; funding acquisition, P.H. and P.W. All authors have read and agreed to the published version of the manuscript.

Funding: This research was funded by the Natural Science Foundation of Hubei Province (China) under Grant no. 2020CFB120, and the National Natural Science Foundation of China under Grant no. 51907144.

Conflicts of Interest: The authors declare no conflict of interest.

\section{References}

1. Wen, T.; Zhu, D.; Zou, X.; Jiang, B.; Peng, L.; Kang, Y. Power coupling mechanism analysis and improved decoupling control for virtual synchronous generator. IEEE Trans. Power Electron. 2021, 36, 3028-3041. [CrossRef]

2. Li, K.; Wen, W.; Zhao, Z.; Yuan, L.; Cai, W.; Mo, X.; Gao, C. Design and Implementation of Four-Port Megawatt-Level HighFrequency-Bus Based Power Electronic Transformer. IEEE Trans. Power Electron. 2021, 36, 6429-6442. [CrossRef]

3. Liu, B.; Wu, W.; Zhou, C.; Mao, C.; Wang, D.; Duan, Q.; Sha, G. An AC-DC hybrid multi-port energy router with coordinated control and energy management strategies. IEEE Access 2019, 7, 109069-109082. [CrossRef] 
4. Ma, D.; Chen, W.; Shu, L.; Qu, X.; Hou, K. A MMC-Based Multiport Power Electronic Transformer With Shared Medium-Frequency Transformer. IEEE Trans. Circuits Syst. II Express Briefs 2021, 68, 727-731. [CrossRef]

5. Gu, C.; Zheng, Z.; Xu, L.; Wang, K.; Li, Y. Modeling and Control of a Multiport Power Electronic Transformer (PET) for Electric Traction Applications. IEEE Trans. Power Electron. 2016, 31, 915-927. [CrossRef]

6. Jafari, M.; Malekjamshidi, Z.; Zhu, J. Accurate copper loss analysis of a multi-winding high-frequency transformer for a magnetically-coupled residential micro-grid. In Proceedings of the 2017 20th International Conference on Electrical Machines and Systems (ICEMS), Sydney, NSW, Australia, 11-14 August 2017; pp. 1-6.

7. Liang, Y.; Wang, Z.; Wu, H.; Wang, C.; Li, X. Design of a multi-winding high-frequency transformer for DC-DC applications. In Proceedings of the 2017 IEEE Conference on Energy Internet and Energy System Integration (EI2), Beijing, China, 26-28 November 2017; pp. 1-6. [CrossRef]

8. Rashidi, M.; Bani-Ahmed, A.; Nasiri, R.; Mazaheri, A.; Nasiri, A. Design and implementation of a multi winding high frequency transformer for MPSST application. In Proceedings of the 2017 IEEE 6th International Conference on Renewable Energy Research and Applications (ICRERA), San Diego, CA, USA, 5-8 November 2017; pp. 491-494.

9. El Shafei, A.; Ozdemir, S.; Altin, N.; Jean-Pierre, G.; Nasiri, A. A Complete Design of a High Frequency Medium Voltage Multi-Port Transformer. In Proceedings of the 2019 8th International Conference on Renewable Energy Research and Applications (ICRERA), Brasov, Romania, 3-6 November 2019; pp. 761-766.

10. Garcia-Bediaga, A.; Villar, I.; Rujas, A.; Mir, L.; Rufer, A. Multiobjective Optimization of Medium-Frequency Transformers for Isolated Soft-Switching Converters Using a Genetic Algorithm. IEEE Trans. Power Electron. 2017, 32, 2995-3006. [CrossRef]

11. Beiranvand, H.; Rokrok, E.; Liseran, M. Vf-constrained $\eta \rho$-pareto optimisation of medium frequency transformers in ISOP-DAB converters. IET Power Electron. 2020, 13, 1984-1994. [CrossRef]

12. Chen, B.; Liang, X.; Wan, N. Design Methodology for Inductor-Integrated Litz-Wired High-Power Medium-Frequency Transformer with the Nanocrystalline Core Material for Isolated DC-Link Stage of Solid-State Transformer. IEEE Trans. Power Electron. 2020, 35, 11557-11573. [CrossRef]

13. Huang, P.; Mao, C.; Wang, D.; Wang, L.; Duan, Y.; Qiu, J.; Xu, G.; Cai, H. Optimal Design and Implementation of High-Voltage High-Power Silicon Steel Core Medium-Frequency Transformer. IEEE Trans. Ind. Electron. 2017, 64, 4391-4401. [CrossRef]

14. Huang, P.; Mao, C.; Wang, D. Analysis of electromagnetic force for medium frequency transformer with interleaved windings. IET Gener. Transm. Distrib. 2017, 11, 2023-2030. [CrossRef]

15. Mogorovic, M.; Dujic, D. $100 \mathrm{~kW}, 10 \mathrm{kHz}$ medium-frequency transformer design optimization and experimental verification. IEEE Trans. Power Electron. 2019, 34, 1696-1708. [CrossRef]

16. Zhang, L.; Zhang, D.; Shui, H.; Yuan, Y.; Pei, Q.; Zhu, J. Optimisation design of medium frequency transformer for the offshore dc grid based on multi-objective genetic algorithm. IET Power Electron. 2017, 10, 2157-2162. [CrossRef]

17. Xu, Y.; Chen, L.; Guo, W.; Shangguan, C.; Zuo, J.; He, K. Optimal Design of Medium-Frequency Fe-Based Amorphous Transformer Based on Genetic Algorithm. IEEE Trans. Plasma Sci. 2018, 46, 3240-3248. [CrossRef]

18. Zhao, Y.; Zhang, G.; Liao, Z.; Wan, L.; Li, Y.; Yang, F. Optimal Design of Insulation Structure of HV-HF Transformer Based on High-Frequency Insulation Properties of Gas-Solid System. In Proceedings of the 2020 IEEE Electrical Insulation Conference (EIC), Knoxville, TN, USA, 22 June-3 July 2020; pp. 482-485.

19. Zhao, S.; Li, Q.; Lee, F.C. High frequency transformer design for modular power conversion from medium voltage AC to 400 VDC. IEEE Trans. Power Electron. 2017, 33, 7545-7557. [CrossRef]

20. Mogorovic, M.; Dujic, D. Sensitivity Analysis of Medium-Frequency Transformer Designs for Solid-State Transformers. IEEE Trans. Power Electron. 2019, 34, 8356-8367. [CrossRef]

21. Tang, T.; Ferreira, J.; Mao, S.; Wang, W.; Niasar, M.G. Design of a Medium Frequency Transformer with High Insulation Level for Dual Active Bridge DC-DC Converter. In Proceedings of the 2019 10th International Conference on Power Electronics and ECCE Asia (ICPE 2019-ECCE Asia), Busan, Korea, 27-30 May 2019; pp. 1-8.

22. Guo, Z.; Yu, R.; Xu, W.; Feng, X.; Huang, A.Q. Design and Optimization of a 200-kW Medium-Frequency Transformer for Medium Voltage SiC PV Inverters. IEEE Trans. Power Electron. 2021, 1. [CrossRef]

23. González, E.; Gómez, P.; Espino-Cortés, F.P. Analysis of the electric field distribution on insulating supports of dry-type transformers under high temperature. IET Electron. Power Appl. 2013, 7, 331-337. [CrossRef]

24. Zhang, B.; Yan, N.; Liu, N. A Novel Method for Thermal Analysis of MF Transformers with Hollow Windings. In Proceedings of the 2019 10th International Conference on Power Electronics and ECCE Asia (ICPE 2019-ECCE Asia), Busan, Korea, 27-30 May 2019; pp. 911-916.

25. Ayat, S.; Daguse, B.; Khazaka, R. Design Considerations of Windings formed with Hollow Conductors Cooled with Phase Change Material. In Proceedings of the 2019 IEEE Energy Conversion Congress and Exposition (ECCE), Baltimore, MD, USA, 29 September-3 October 2019; pp. 5652-5658.

26. Chen, X.; Wang, J.; Griffo, A.; Spagnolo, A. Thermal Modeling of Hollow Conductors for Direct Cooling of Electrical Machines. IEEE Trans. Ind. Electron. 2019, 67, 895-905. [CrossRef]

27. Yuan, F.; Yang, S.; Qin, S.; Tang, B.; Lv, K.; Han, S.; Ding, C.; Huang, L. The Optimization Design of Sound Arrester for the Dry Type Air Core Smoothing Reactor Based on the Multi-Physical Field Coupling Method. IEEJ Trans. Electr. Electron. Eng. 2021, 16, 704-714. [CrossRef] 
28. Faiz, J.; Ebrahimi, B.M.; Noori, T. Three- and Two-Dimensional Finite-Element Computation of Inrush Current and Short-Circuit Electromagnetic Forces on Windings of a Three-Phase Core-Type Power Transformer. IEEE Trans. Magn. 2008, 44, 590-597. [CrossRef]

29. Yin, K. Transformer Design Principle; China Electric Power Press: Beijing, China, 2003; pp. 37-38. 